\title{
Comparison of the Hypersensitive Response Induced by the Tomato $C f-4$ and $C f-9$ Genes in Nicotiana spp.
}

\author{
Colwyn M. Thomas, ${ }^{1}$ Saijun Tang, ${ }^{2}$ Kim Hammond-Kosack, ${ }^{3}$ and Jonathan D. G. Jones ${ }^{1}$ \\ ${ }^{1}$ Sainsbury Laboratory, John Innes Centre, Norwich Research Park, Colney Lane, Norwich NR4 7UH, U.K.; \\ ${ }^{2}$ Department of Biology, 108 Coker Hall CB 3280, University of North Carolina, Chapel Hill 27599-3280, \\ U.S.A.; ${ }^{3}$ Monsanto PLC, Cereal Technology Group, Maris Lane, Trumpington, Cambridge, CB2 2LQ, U.K. \\ Accepted 2 December 1999.
}

\begin{abstract}
We have previously shown that tomato $C f-9$ induces an Avr9-dependent hypersensitive response (HR) in Nicotiana tabacum and potato. We show here that $C f-4$ also induces an $A v r 4$-dependent HR in two tobacco species ( $N$. tabacum and $N$. benthamiana). The HR induced by $C f-4$ and $C f-9$ was compared in stable tobacco transgenics by a seedling lethal assay and resistance to recombinant Potato virus $\mathbf{X}$ expressing Avr4 or Avr9. We also compared HR induction with Agrobacterium-mediated transient expression. The $C f-4 / A v r 4$ combination induced a more rapid HR than $C f$ 9/Avr9. Sensitive assays for $\boldsymbol{C f}-9$ and $\boldsymbol{C f}$-4 function should prove useful for structure/function analyses of these resistance proteins in tobacco.
\end{abstract}

Additional keywords: $C f$ genes, disease resistance.

Tomato $C f$ genes encode extracellular membrane anchored leucine-rich repeat (LRR) glycoproteins that confer resistance to Cladosporium fulvum through recognition of distinct fungal avirulence (Avr) determinants (Thomas et al. 1998). DNA sequence analysis of homologous genes from a number of chromosomal loci have identified LRR sequences that determine recognition specificity (Dixon et al. 1998; Parniske et al. 1997; Thomas et al. 1997). Functional analysis of these proteins requires the development of robust assays for $\mathrm{Cf}$ activity. The genes for two C. fulvum Avr determinants (Avr4 and Avr9) have been cloned (Joosten et al. 1994; Van den Ackerveken et al. 1992) and are useful reagents to study Cf-4 and Cf-9 function in tomato. We have previously shown that $C f-9$ induces an Avr9-dependent hypersensitive response (HR) in Nicotiana tabacum (Hammond-Kosack et al. 1998). Here we describe sensitive assays to analyze the HR-inducing activity of $C f-9$ and $C f-4$ in tobacco.

Progeny from $N$. tabacum $C f-9$ plants crossed to $N$. tabacum 35S:Avr9 lines show a seedling lethal phenotype (Hammond-Kosack et al. 1998). To compare $C f$-9- and $C f$-4mediated seedling lethality in tobacco, binary vectors were constructed that expressed $C f-4$ or $C f-9$ from the $C f-4$ promoter. This element consisted of $3.0 \mathrm{~kb}$ of DNA upstream of $C f-4$ from plasmid p129P6 (Thomas et al. 1997). These con-

Corresponding author: J. D. G. Jones; Telephone: + 441603 452571; Fax: + 441603 250024; E-mail: jonathan.jones@bbsrc.ac.uk structs were used to transform N. tabacum cv. Petite Gerard (C. M. Thomas, M. Smoker, and J. D. G. Jones, manuscript in preparation). N. tabacum lines expressing 35S:Avr4 (Thomas et al. 1997) were also generated. Six primary $C f-4$ and $C f-9$ transgenics were crossed reciprocally to $35 \mathrm{~S}: A v r 4$ or $35 \mathrm{~S}: A v r 9$ (Hammond-Kosack et al. 1998) transgenic lines. Four transgenic lines carrying each construct that contained single TDNA loci were analyzed further.

All progeny from crosses between $C f-4$ primary transformants and a line homozygous for 35S:Avr4 germinated and segregated 1:1 for wild type and a seedling lethal phenotype irrespective of which plant was used as male or female parent (Fig. 1). PCR (polymerase chain reaction) analysis confirmed that wild-type seedlings inherited only 35S:Avr4. Progeny from crosses between $C f-9$ primary transformants crossed as male parent to a $35 \mathrm{~S}: A v r 9$ homozygous line as female parent also segregated 1:1 for wild-type and necrotic seedlings, as described previously (Hammond-Kosack et al. 1998). The $C f$ 4/Avr4-dependent seedling lethal phenotype was distinct from that observed in $C f-9 / A v r 9$ seeds. Seedlings from the latter cross showed cotyledon expansion and appeared fully green before the onset of necrosis that could be observed initially at the margins of the cotyledons (Fig. 1D). In progeny expressing both $C f-4$ and Avr4, a more severe seedling lethal phenotype was observed. Cotyledons showed reduced expansion to the extent that the testa remained attached, and were never green (Fig. 1B). Therefore, the seedling lethal phenotype induced by $C f-4 / A v r 4$ in tobacco appears to act earlier in development than that induced by $C f-9 / A v r 9$.

In this study, progeny from crosses between $C f-9$ primary transformants crossed as female parent to a $35 \mathrm{~S}: A v r 9$ homozygous line as male parent segregated 1:1 for wild type and a more severe non-germination phenotype (results not shown). This result contrasts with a previous study in which $C f-9$ was expressed from its own promoter (Hammond-Kosack et al. 1998). In that study, nearly $100 \%$ of the progeny from crosses with $N$. tabacum $C f-9$ heterozygotes, crossed as female parents to the same 35S:Avr9 homozygous line used here, failed to germinate (Hammond-Kosack et al. 1998). The seed nonautonomous phenotype was postulated to be due to diffusion of the 28 amino acid Avr9 peptide from zygotic tissue into surrounding maternal tissue that expressed $C f-9$ and triggered a cellular response mediated by salicylic acid. The different 
results may be due to the different promoters used, but DNA sequencing has shown the $C f-9$ and $C f-4$ upstream sequences are highly homologous (Parniske et al. 1997). It is also unlikely that this is due to differences in the binary vectors used since in each case $C f$ transgenes were cloned in near-identical vectors (SLJ 7291 and SLJ 7292; Jones et al. 1992). Cf-9 expression in the two transgenics studied previously may have been elevated due to additional sequences present on the 4.5kb sequence upstream of $C f-9$ (Hammond-Kosack et al. 1998), the orientation of $C f$ genes within the T-DNA, or their chromosomal location.

Elevated levels of $C f-4$ expression also result in a seed nongermination phenotype. In progeny from crosses between $N$. tabacum 35S:Cf-4 heterozygotes and plants heterozygous for $35 \mathrm{~S}: A v r 4$, one quarter of the progeny failed to germinate, indicating the phenotype is seed autonomous (results not shown). This phenotype was not dependent upon which plant was used as male or female parent. The seed autonomy in 35S:Cf-4 $\times 35 \mathrm{~S}: A v r 4$ crosses may be due to the larger size of Avr4 (106 amino acids; Thomas et al. 1997), which may be less diffusible than Avr9, or alternatively Avr4 may be more labile.

To test whether Agrobacterium tumefaciens could be used to transiently express $C f$ - 9 to induce an $A v r 9$-dependent HR, binary vectors were constructed based on a $C f-9$ cDNA clone (Jones et al. 1994; Tang 1998). In one vector, $C f-9$ expression was under the control of the $35 \mathrm{~S}$ promoter and the A. tumefaciens nopaline synthase $3^{\prime}$ terminator (nos $\left.3^{\prime}\right)$. In a similar vector, which served as a negative control, transcription of $C f$ 9 was interrupted by the maize transposon Activator inserted between $35 \mathrm{~S}$ and $C f-9$. Bacteria were infiltrated into leaves of N. tabacum 35S:Avr9 transgenic plants (Fig. 2). Necrosis was observed in leaf panels infiltrated with A. tumefaciens expressing 35S:Cf-9 at 7 days post infiltration (Fig. 2A). No necrosis was observed in leaf panels expressing empty vector sequences or $35 \mathrm{~S}: A c: C f-9$. These results demonstrate that Agrobacterium-mediated transient expression of 35S:Cf-9 can induce an Avr9-dependent HR. In addition, no macroscopic HR could be detected in leaf panels infiltrated with a 35S: $C f$ -
9 $\mathrm{MA}$ construct that lacks domains $\mathrm{E}, \mathrm{F}$, and $\mathrm{G}$ of Cf-9 (Fig. 2A). This shows that Cf-9 requires a functional juxtamembrane, transmembrane, or cytoplasmic domain, or alternatively all three domains, to induce an Avr9-dependent HR.

To compare the HR-inducing activity of $C f-4$ and $C f-9$, additional vectors were made that co-expressed $C f$ and $A v r$ gene cassettes from identical transcription control sequences (Fig. 2; Jones et al. 1995). Binary vectors were mobilized into $A$. tumefaciens GV3101. The HR in N. benthamiana was induced more rapidly than in $N$. tabacum. Necrosis was observed in $N$. benthamiana leaf panels expressing $C f-4$ or $C f-9$ only in the presence of the corresponding avirulence determinant (Fig. 2B and $\mathrm{C}$ ). The necrotic reaction induced by $C f-4 / A v r 4$ was fully developed after 4 days, compared with the HR induced by $C f$ $9 / A v r 9$, which was fully developed after 7 days. This result is consistent with data from seedling lethal assays that suggested $C f-4 / A v r 4$ induces a more rapid HR.

Tobacco transgenics expressing $C f-9$ are resistant to Potato virus $X$ (PVX) infection when it carries the corresponding Avr9 avirulence determinant (Kamoun et al. 1999), as also shown for tomato expressing Pto after inoculation with PVX:avrpto (Tobias et al. 1999). This contrasts with the phenotypes observed in tomato Cf9 and Cf4 lines where PVX expressing the corresponding $A v r$ gene replicates, resulting in systemic necrosis and plant death (Hammond-Kosack et al. 1995; Thomas et al. 1997). The relative effectiveness of $N$. tabacum $C f-4$ and $C f-9$ transgenic lines in resisting infection to PVX:Avr4 (Thomas et al. 1997) and PVX:Avr9 (HammondKosack et al. 1995) was determined.

Equal titers of wild-type PVX, PVX:Avr4, and PVX:Avr9 were inoculated onto $N$. tabacum transgenics at the six- to eight-leaf stage. The three PVX derivatives were all compatible on N. tabacum control plants (Fig. 3A) and produced typical symptoms of PVX infection in systemically infected leaves (vein clearing, chlorosis, and mosaic symptoms). The yield of recombinant virus in compatible reactions was reduced, compared with wild-type PVX, particularly PVX:Avr4 (Fig. 3). In inoculated and upper leaves of PVX:Avr4-infected plants, the PVX RNA was heterogeneous and two major
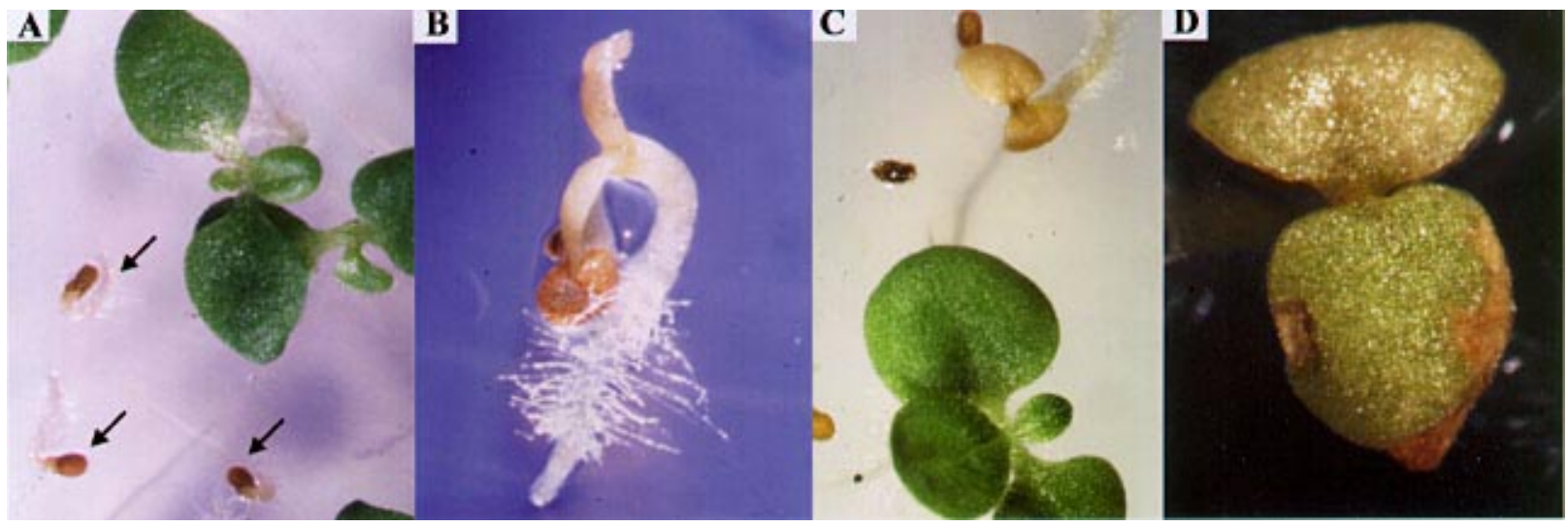

Fig. 1. Seedling lethal phenotype in transgenic Nicotiana tabacum expressing $C f-4$ or $C f-9$ from the $C f-4$ promoter. Seedlings were photographed 18 days post sowing (dps) except in D. A, Progeny from a cross between a plant hemizygous for $C f-4$ (F641) crossed to a $35 \mathrm{~S}$ :Avr4 homozygote. Seedlings expressing both transgenes (indicated by arrows) show retarded root development and limited cotyledon expansion and many retain the testa. B, In some $C f-4 / A v r 4$ seedlings expansion of the cotyledons was observed at $18 \mathrm{dps}$ but they were devoid of chlorophyll. C, Seedlings from a cross between a transformant hemizygous for $C f-9$ (F797) crossed to a 35S:Avr9 homozygous line. Seedlings expressing $C f-9$ and $A v r 9$ were initially green as previously reported. D, Tobacco seedling expressing $C f-9$ and $A v r 9$ at 13 dps. After development of fully green cotyledons, necrotic sectors were observed initially at the margins of the cotyledons. 
bands corresponding to recombinant and wild-type virus RNA were detected (Fig. 3). In contrast, PVX:Avr9 appeared more stable (Fig. 3).

Incompatible reactions were observed when recombinant PVX was inoculated onto transgenic $N$. tabacum expressing the corresponding $C f$ gene (Fig. 3B and C). Necrotic lesions were observed on inoculated leaves 4 to 5 days post inoculation and plants failed to develop typical symptoms of PVX infection. The absence of virus in these plants was confirmed by RNA gel blot analysis (Fig. 3B and C) and by inoculating sap extracted from these leaves onto $N$. benthamiana plants that are highly susceptible to PVX infection. The local lesion

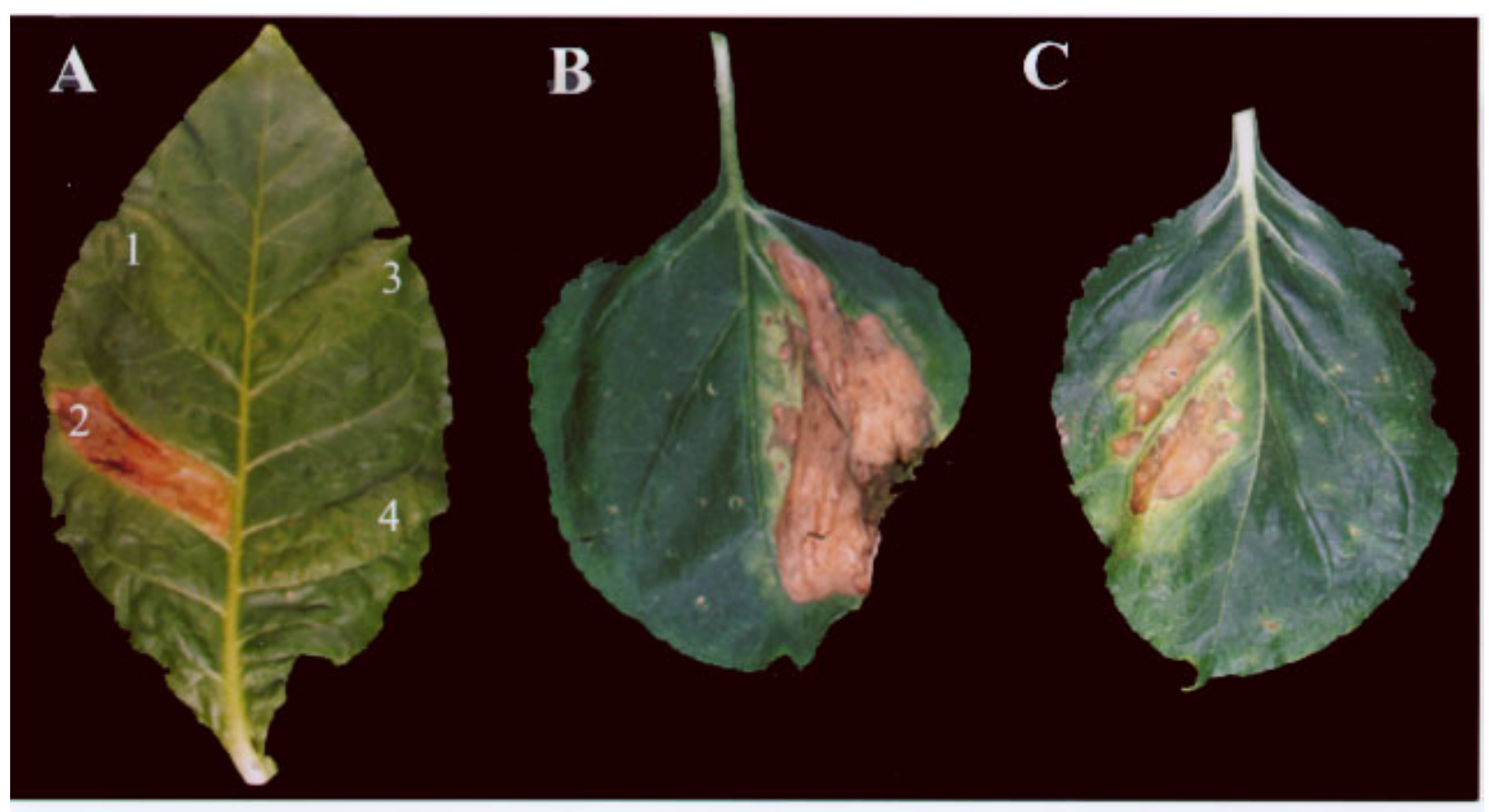

D

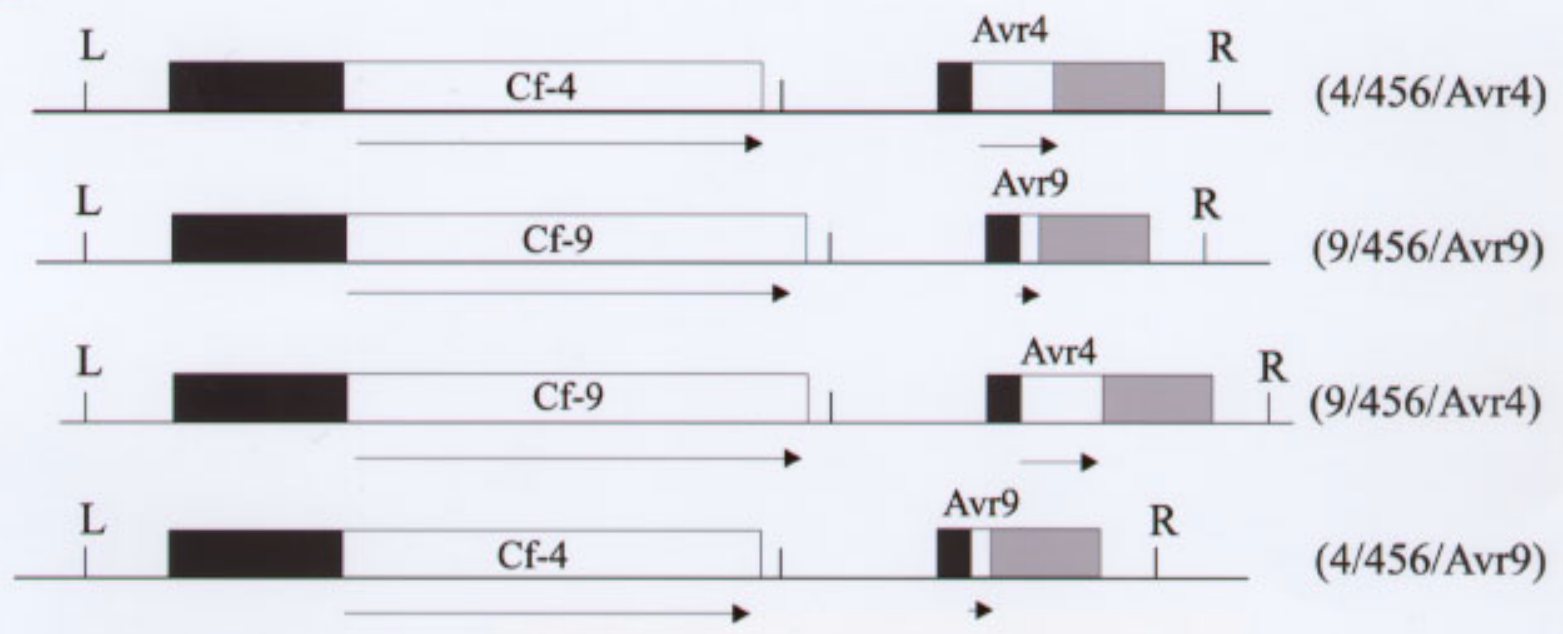

Fig. 2. Expression of $C f-4$ and $C f-9$ in tobacco with Agrobacterium-mediated transient expression. Constructs were mobilized into A. tumefaciens GV3101 by tri-parental mating. A. tumefaciens was resuspended at an $\mathrm{OD}_{600}$ of 0.5 in Murashige and Skoog salts, $2 \%$ wt/vol sucrose, $500 \mu \mathrm{M} 2-(N-$ morpholino)ethane sulfonic acid (MES) pH 5.6 and $20 \mu \mathrm{M}$ acetosyringone. Bacteria were incubated at $22^{\circ} \mathrm{C}$ for $3 \mathrm{~h}$ without shaking then infiltrated into leaves of Nicotiana tabacum 35S:Avr9 with a syringe. Plants were maintained in a glasshouse at $22^{\circ} \mathrm{C}$ with a 16 -h photoperiod supplied at a photon flux density of 300 to $650 \mu \mathrm{E} \mathrm{s}^{-1} \mathrm{~m}^{-2}$. A, $C f-9$ expression in infiltrated leaf panels of $N$. tabacum 35S:Avr 9 at 7 days post infiltration. Leaf panels were infiltrated with A. tumefaciens GV3101 containing empty vector (SLJ 7291, panel 1), 35S:Cf-9 (SLJ 8381, panel 2), 35S:Ac:Cf-9 (SLJ 8511, panel 3), and 35S:Cf-9 $\mathrm{MA}$ (SLJ 8407, panel 4). B, Analysis of $C f-4 / A v r$ co-expression constructs in wild-type $N$. benthamiana at 5 days post infiltration. Leaves were injected with $C f-4 / A v r 9$ (4/456/Avr9, left hand panel) and $C f$-4/Avr4 (4/456/Avr4, right hand panel). C, Analysis of $C f$-9/Avr co-expression constructs at 9 days post infiltration; leaves were infiltrated with $C f-9 / A v r 9$ (9/456/Avr9, left hand panel) or $C f-9 / A v r 4$ (9/456/Avr4, right hand panel). D, Binary vector constructs for co-expression of $C f$ and $A v r$ genes. $C f$ gene expression cassettes comprised $C f-4$ and $C f$ - 9 genomic sequences including their identical 3' untranslated regions that contain a small intron (Thomas et al. 1997). Expression was under control of the $35 \mathrm{~S}$ pro moter. Avr4 and Avr9 expression cassettes contained the $35 \mathrm{~S}$ promoter and the nos $3^{\prime}$ terminator. $35 \mathrm{~S}$ sequences are shown as black boxes and the nos $3^{\prime}$ terminator sequence is shown in gray. $C f$ and $A v r$ sequences are shown as white boxes, $\mathrm{L}$ and $\mathrm{R}$ correspond to the left and right border T-DNA sequences. 

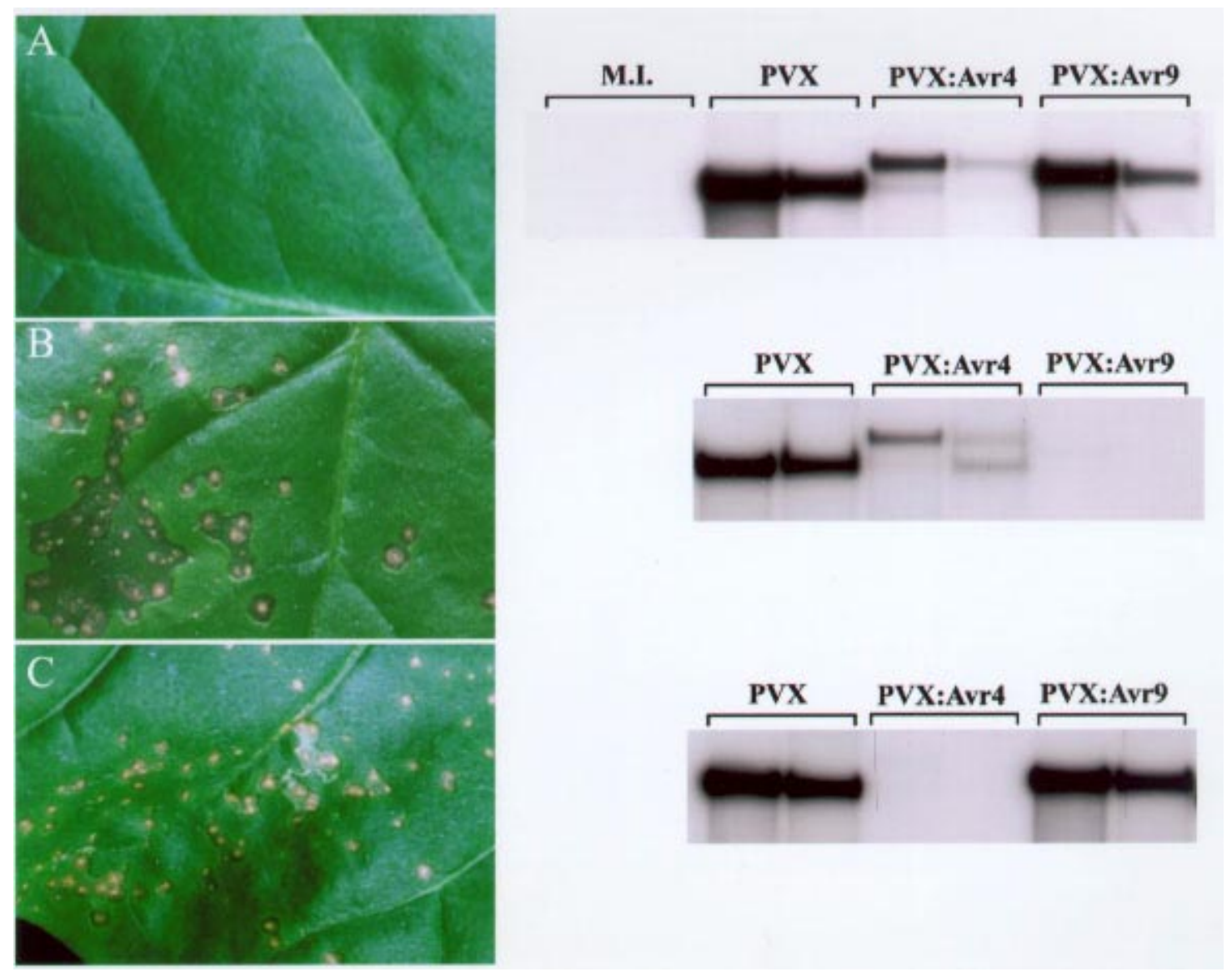

Fig. 3. $C f-4$ - and $C f-9$-mediated resistance in Nicotiana tabacum to recombinant Potato virus $X$ (PVX). A, Leaf of nontransformed $N$. tabacum cv. Petite Gerard inoculated with PVX at 5 days post inoculation (dpi). Adjacent panel shows an RNA gel blot with ${ }^{32} \mathrm{P}-$ labeled PVX cDNA used as a probe. Ten micrograms of total RNA (equivalent RNA loading was confirmed by ethidium bromide staining) was isolated from plants inoculated with PVX, PVX:Avr4, PVX:Avr9, or mock-inoculated (M.I.) plants. Total RNA was prepared from inoculated and "systemically infected" leaves (three uppermost leaves) at 10 dpi. In the gel blot, the first lane contains RNA from inoculated leaves; the second lane RNA from "systemically infected" leaves. Only the region of the blot containing full-length PVX RNA is shown. B, Local lesion phenotype induced by PVX:Avr 9 at 5 dpi in a transgenic line heterozygous for $C f-9$ transgenic (F797) and corresponding RNA gel blot from siblings inoculated with PVX and PVX:Avr4. Local lesions induced by $C f-9$ in incompatible reactions eventually merged to produce a necrotic sector (data not shown). C, Local lesion phenotype induced by PVX:Avr4 in a transformant heterozygous for $C f-4$ (F640) and RNA gel blot of samples isolated at 10 dpi. Lesions induced by $C f-4$ in incompatible reactions were always smaller than those observed in $C f-9$ transformants and no spreading necrosis was observed.

phenotypes in incompatible reactions induced by $C f-9$ and $C f$ 4 transgenics were distinct. Larger necrotic lesions were observed in $C f-9$ plants inoculated with PVX:Avr9 that spread and eventually coalesced to generate a necrotic leaf sector (Fig. 3). The spreading $C f$-9/Avr9-dependent necrosis was reported previously in response to Avr9 infiltration into N. tabacum $C f-9$ leaves and is potentiated by salicylic acid (Hammond-Kosack et al. 1998). The lesions induced by PVX:Avr4 on N. tabacum Cf-4 leaves were smaller in the four independent transgenics tested and remained discrete. The smaller lesions induced by $C f-4$ are probably due to the slower rate of PVX:Avr4 replication and the more rapid HR induced by $C f-4 / A v r 4$ in tobacco.

Tobacco is a useful system in which to study the Cf/Avrinduced HR at biochemical, physiological, and molecular levels (Piedras et al. 1998; Romeis et al. 1999). We have developed a number of sensitive assays to monitor induction of the HR in tobacco. Our analysis showed that $C f-4 / A v r 4$ induces a more rapid HR than $C f-9 / A v r 9$. These assays, particularly Agrobacterium-mediated transient expression, should facili- tate a structure/function analysis of Cf-4 and Cf-9 in tobacco. Characterization of HR induction by specific resistance gene/ Avr gene combinations is an important criterion for engineering resistance in heterologous species (De Wit 1992). Therefore, in strategies for engineering disease resistance in solanaceous plants (Hammond-Kosack et al. 1998), the Cf-4/Avr4 combination that induces a rapid HR may prove most effective in arresting pathogen ingress.

\section{ACKNOWLEDGMENTS}

Research in the Sainsbury Laboratory is funded by the Gatsby Charitable foundation. S. T. was in receipt of a Gatsby Ph.D. studentship. We are grateful to Sara Perkins and Justine Campling for plant care and maintenance.

\section{LITERATURE CITED}

De Wit, P. J. G. M. 1992. Molecular characterization of gene-for-gene systems in plant-fungus interactions and the application of aviru- 
lence genes in control of plant pathogens. Annu. Rev. Phytopathol. 30:391-418

Dixon, M. S., Hatzixanthis, K., Jones, D. A., Harrison, K., and Jones, J. D. G. 1998. The tomato Cf-5 disease resistance gene and six homologs show pronounced allelic variation in leucine-rich repeat copy number. Plant Cell 10:1915-1925.

Hammond-Kosack, K. E., Staskawicz, B. J., Jones, J. D. G., and Baulcombe, D. C. 1995. Functional expression of a fungal avirulence gene from a modified potato virus X genome. Mol. Plant-Microbe Interact. 8:181-185.

Hammond-Kosack, K. E., Tang, S., Harrison, K., and Jones, J. D. G. 1998. The tomato $C f-9$ disease resistance gene functions in tomato and potato to confer responsiveness to the fungal avirulence gene product Avr9. Plant Cell 10:1251-1266.

Jones, J. D. G., Shlumukov, L., Carland, F. J., Scofield, S., Bishop, G., and Harrison, K. 1992. Effective vectors for transformation, expression of heterologous genes, and assaying transposon excision in transgenic plants. Transgenic Res. 1:285-297.

Jones, J. D. G., Thomas, C. M., Balint-Kurti, P. J., and Jones, D. A. 1995. Plant pathogen resistance genes and uses thereof. U.K. patent WO96/35790.

Jones, D. A., Thomas, C. M., Hammond-Kosack, K. E., Balint-Kurti, P. J., and Jones, J. D. G. 1994. Isolation of the tomato $C f-9$ gene for resistance to Cladosporium fulvum by transposon tagging. Science 266: 789-793.

Joosten, M. H. A. J., Cozijnsen, T. J., and De Wit, P. J. G. M. 1994. Host resistance to a fungal tomato pathogen lost by a single base-pair change in an avirulence gene. Nature 367:384-386.

Kamoun, S., Honée, G., Weide, R., Laugé, R., Kooman-Gersmann, M., de Groot, K., Govers, F., and De Wit, P. J. G. M. 1999. The fungal gene Avr 9 and the oomycete gene infl confer avirulence to potato virus X on tobacco. Mol. Plant-Microbe Interact. 12:459-462.
Parniske, M., Hammond-Kosack, K. E., Golstein, C., Thomas, C. M., Jones, D. A., Harrison, K., Wulff, B. B. H., and Jones, J. D. G. 1997. Novel disease resistance specificities result from sequence exchange between tandemly repeated genes at the $C f-4 / 9$ locus of tomato. Cell 91:821-832.

Piedras, P., Hammond-Kosack, K. E.. Harrison, K., and Jones, J. D. G. 1998. Rapid, Cf9- and Avr9-dependent production of active oxygen species in tobacco suspension cultures. Mol. Plant-Microbe Interact. 11:1155-1166.

Romeis, T., Piedras, P., Zhang, S., Klessig, D. F., Hirt, H., and Jones, J. D. G. 1999. Rapid Avr9- and Cf-9-dependent activation of MAP kinases in tobacco cell cultures and leaves: convergence of resistance gene, elicitor, wound and salicylate responses. Plant Cell 11:273-287.

Tang, S. 1998. Analysis of tomato $C f$ gene function. Ph.D. thesis. University of East Anglia, Norwich, UK.

Thomas, C. M., Dixon, M. S., Parniske, M., Golstein. C., and Jones, J. D. G. 1998. Genetic and molecular analysis of tomato $C f$ genes for resistance to Cladosporium fulvum. Phil. Trans. R. Soc. Lond. B 353: $1413-1424$

Thomas, C. M., Jones, D. A., Parniske, M., Harrison, K., Balint-Kurti, P. J., Hatzixanthis, K., and Jones, J. D. G. 1997. Characterization of the tomato $C f-4$ gene for resistance to Cladosporium fulvum identifies sequences that determine recognitional specificity in Cf- 4 and Cf- 9 . Plant Cell 9:2209-2224.

Tobias, C. M., Oldroyd, G. E. D., Chang, J. H., Staskawicz, B. J. 1999. Plants expressing the Pto disease resistance gene confer resistance to recombinant PVX containing the avirulence gene AvrPto. Plant J. 17:41-50.

Van den Ackerveken, G. F. J. M., Van Kan, J. A. L., and De Wit, P. J. G. M. 1992. Molecular analysis of the avirulence gene avr9 of the fungal tomato pathogen Cladosporium fulvum fully supports the gene-forgene hypothesis. Plant J. 2:359-366. 